\title{
ESTRÉS ACADÉMICO EN ESTUDIANTES UNIVERSITARIOS FRENTE A LA EDUCACIÓN VIRTUAL ASOCIADA AL COVID-19
}

\author{
ACADEMIC STRESS IN UNIVERSITY STUDENTS IN THE FACE OF \\ VIRTUAL EDUCATION ASSOCIATED WITH COVID-19
}

Olivia Magaly Luque Vilca ${ }^{*}$, Nestor Bolivar Espinoza ${ }^{1}$, Victor Ernesto Achahui Ugarte ${ }^{1}$, Julio Rumualdo Gallegos Ramos ${ }^{1}$

oluque@unaj.edu.pe; nbolivar@unaj.edu.pe; v.achahui@unaj.edu.pe; jgallegos@unaj.edu.pe

${ }^{1}$ Universidad Nacional de Juliaca, Puno, Perú

*Correspondencia: Olivia Magaly Luque Vilca. Email: oluque@unaj.edu.pe

Recibido: 06.06.21 | Aprobado: 20.06.21

\section{RESUMEN}

El estrés académico en estudiantes universitarios asociado a la pandemia COVID19 está incrementándose debido al cambio de la educación presencial a virtual, causando disminución de motivación y aumento de ansiedad en los estudiantes. El objetivo del presente trabajo, es la evaluación del estrés académico en estudiantes universitarios frente a la educación virtual asociada al COVID-19, para ello se aplicó el cuestionario validado de estrés académico SISCO SV- COVID-19, obteniendo que el $92.27 \%$ de estudiantes encuestados tienen estrés académico, identificándose como factor estresor a la sobrecarga de tareas y trabajos que deben cumplir en los plazos establecidos por los docentes.

Palabras claves: COVID-19, estrés académico, educación virtual, factor estresor.

\begin{abstract}
Academic stress in university students associated with the COVID-19 pandemic is increasing due to the change from face-to-face to virtual education, causing decreased motivation and increased anxiety in students. The aim of this study is to evaluate academic stress in university students in the face of virtual education associated with COVID-19. To this end, the validated academic stress questionnaire SISCO SV-COVID-19 was applied, and $92.27 \%$ of the students surveyed had academic stress, identifying the overload of tasks and work to be completed within the deadlines set by teachers as a stress factor.
\end{abstract}

Keywords: COVID-19, academic stress, eating habits and performance. 


\section{INTRODUCCIÓN}

La educación superior, está pasando por un proceso de cambio y adaptación de la forma de trasmitir conocimientos, esto debido a las condiciones actuales de pandemia COVID-19 que surgió a principios de diciembre de 2019 y que posteriormente se ha extendido por todo el mundo (Basturk et al., 2020) afectando directamente a docentes y estudiantes con el estrés académico. Según UNESCO (IELSAC, 2020) en América Latina el confinamiento inició en marzo del 2020 y Perú no fue la excepción, esta medida afectó a las Instituciones de Educación Universitaria porque no tenían las medidas de contingencia para continuar con la educación a distancia, afectando a los estudiantes en la interrupción de actividades académicas (Baptista Lucio et al., 2020), pasando por un proceso de adaptación reorganizando su vida cotidiana, a la pérdida de contacto social y al aislamiento. En este contexto para dar continuidad a las actividades académicas se adecuó el proceso de enseñanza y aprendizaje a la modalidad virtual mediante la implementación de un plan de estudios virtuales (Gelineau-Morel \& Dilts, 2021), a fin de mitigar la pandemia COVID-19, que es necesaria desde el punto de vista de la salud pública; por lo que los docentes y estudiantes se enfrentaron ante esta situación conllevando a cambios drásticos, aumentando los niveles de estrés y ansiedad durante la pandemia (Husky et al., 2020) y algunas personas que padecen de algunas enfermedades tienen que tomar en cuenta y ajustar su tratamiento a estas condiciones (Khosravani et al., 2021).

Ante esta situación es probable que se produzcan consecuencias psicológicas negativas en las personas a causa de la presión psicológica en todo el mundo (Ye et al., 2020), causando estrés, que es un conjunto de procesos y respuestas neuroendocrinas, inmunológicas, emocionales y conductuales ante situaciones que significan una mayor adaptación que lo habitual para el organismo, y/o son percibidas por el individuo como amenaza (Berrío García \& Mazo Zea, 2011). Así también, el continuar con estudios universitarios significa propensión a desarrollar nivel de estrés que puede desencadenar problemas emocionales, cognitivos y fisiológicos (Toribio Ferrer \& Franco Bárcenas, 2016), y asociado al cambio de modalidad de enseñanza, los estudiantes se enfrentarse ante el estrés académico y experimentan cansancio, poco interés frente al estudio, nerviosismo e incluso pérdida de control; provocando alteración del sueño, evitación de la responsabilidad y otras manifestaciones que resulten negativamente en el desempeño de su labor como futuros profesionales y en el logro de sus aspiraciones personales (Berrío García \& Mazo Zea, 2011).

Por otro lado los conflictos familiares, las pocas horas de sueño, el género femenino y el bajo rendimiento académico se relacionan a mayores niveles de estrés (SimonelliMuñoz et al., 2018) y sobre todo en estudiantes de menor nivel socioeconómico (Gonzalez, 2020) que son más predispuestos a los factores de riesgo que promueven la deficiencia del sueño, el sobrepeso o el estrés académico, por ejemplo, ingesta de alimentos poco saludables, alto consumo de medios o la pérdida de estrategias de afrontamiento del estrés académico (Buzek et al., 2019), por lo que es necesario la formulación e integración de programas de asesoramiento y manejo del estrés en línea ayudaría a mitigar el estrés de los estudiantes durante el aprendizaje a distancia (AlAteeq et al., 2020), a fin que los estudiantes afronten esta problemática, el objetivo de la presente 
investigación fue evaluar los niveles de estrés académico en estudiantes universitarios frente a la educación virtual asociada al COVID-19.

\section{MATERIALES Y MÉTODOS PARTICIPANTES}

En la presente investigación se ha considerado una población estudiantil conformada por 347 estudiantes universitarios de la Escuela Profesional de Ingeniería en Industrias Alimentarias de la Universidad Nacional de Juliaca, matriculados en el Semestre Académico 2020-II, de una edad media de 22 años, con un rango que va de 16 - 30 años, obteniendo una muestra probabilística de 194 estudiantes con $99 \%$ de confianza y $4.15 \%$ de error de muestreo, la recolección de datos se hizo durante el mes de diciembre del 2020, de los participantes 99 son mujeres $(56.82 \%)$ y 95 varones $(43.18 \%)$, considerando que el tamaño de muestra permite a los investigadores saber cuántos individuos son necesarios estudiar, para estimar el grado de confianza deseado (Karim et al., 2013) .

Los datos fueron recolectados mediante enlace virtual dando a conocer el objetivo del estudio y el consentimiento informado. La participación fue voluntaria, asegurando el resguardo de la identidad y la confidencialidad ya que la información recolectada sólo se utilizará para fines académicos.

\section{INSTRUMENTOS}

Para medir el estrés académico se utilizó el cuestionario de estrés académico SISCO SV al contexto de la crisis por COVID-19, que tiene validez genérica y específica de contenido, con coeficientes de concordancia V de Aiken mayores a 0.75; el Inventario de Estrés Académico SISCO SV adaptado al contexto de la crisis por COVID-19 tiene validez de constructo, con coeficientes de correlación $r$ de Pearson corregida mayores a 0.2; el Inventario de Estrés Académico SISCO SV adaptado al contexto de la crisis por COVID-19 es confiable, con coeficientes de consistencia interna alfa de Cronbach superiores a 0.88 (Alania-Contreras et al., 2020), que consta de 47 items, con dimensiones: de estresores, síntomas y estrategias de afrontamiento.

\section{ANÁLISIS DE DATOS}

Para el análisis de los resultados se utilizó estadística descriptiva que permitió analizar el comportamiento de la distribución en relación a sus valores de tendencia central, efectuando una prueba no paramétrica debido al nivel de escala ordinal de las dimensiones: estresores, síntomas y estrategias de afrontamiento. Se analizó cada dimensión, elaborado por Barraza A., (2007), medidos mediante la escala ordinal de likert: Nunca (1), Casi nunca (2), Rara vez (3), Algunas veces (4), Casi siempre (5) y Siempre (6). Las categorías 1, 2 y 3 denotan ítems de baja o contribución al estrés académico.

\section{RESULTADOS Y DISCUSIONES}

De la población total el 56.82\% correspondió a estudiantes mujeres y el $43.18 \%$ a varones, lo cual es representativo de la población total de estudiantes de la Escuela Profesional de Ingeniería en Industrias Alimentarias de la Universidad Nacional de Juliaca. Del total de estudiantes, el $92.27 \%$ señala haber presentado estrés académico, existiendo una diferencia entre géneros, teniendo en cuenta que el 95\% del género femenino presenta niveles elevados de estrés en comparación al género masculino, considerando que el estrés 
académico es un tipo de estrés cuya fuente se encuentra en el ámbito educativo, y el modelo sistémico cognoscitivista que explica cómo una serie de procesos valorativos ante estímulos estresores del entorno, que tienen el objetivo de lograr un equilibrio sistémico de la relación persona-entorno (Berrío García \& Mazo Zea, 2011). Al observar la Tabla 01 se puede encontrar los factores que son valorados por estudiantes como estresores.

Los factores estresores más frecuentes en los estudiantes es la sobrecarga de tareas, trabajos que tienen que realizar diariamente (3.66), resultado similar en estudios realizado en estudiantes universitarios de las carreras del área de la salud de la Universidad de Los Lagos de Osorno (Jerez-Mendoza \& Oyarzo-Barría, 2015); el tiempo limitado que otorga el docente para la presentación de los mismos (3.22) y la forma de evaluación (3.13) resultado similar en estudios realizados donde la carga de exámenes, así como la falta de tiempo de ocio durante el semestre, se han señalado como determinantes del estrés académico (Ahmad et al., 2019); mientras el tipo de trabajo que piden los docentes (3.09) y la realización de exámenes, prácticas entre otros (3.5), resultado que concuerda con estudios nacionales e internacionales en relación a la alta prevalencia de estrés en los estudiantes universitarios; siendo los factores menos estresantes "que mis profesores están mal preparados" y "la competitividad con o entre mis compañeros de clase".

\section{Tabla 01}

\section{Media de factores estresores}

\begin{tabular}{|c|c|c|}
\hline ITEMs & Media & $\begin{array}{l}\text { Desviación } \\
\text { estándar }\end{array}$ \\
\hline $\begin{array}{l}\text { La sobrecarga de tareas y trabajos académicos que tengo que realizar todos los } \\
\text { días. }\end{array}$ & 3.66 & 1.133 \\
\hline Tener tiempo limitado para hacer el trabajo que me encargan mis profesores/as. & 3.22 & 1.317 \\
\hline $\begin{array}{l}\text { La forma de evaluación de los profesores/as de las tareas, foros, proyectos, } \\
\text { lecturas, ensayos, trabajos de investigación, organizadores, búsquedas en Internet, } \\
\text { etc. }\end{array}$ & 3.13 & 1.335 \\
\hline $\begin{array}{l}\text { El tipo de trabajo que me piden mis profesores/as (análisis de lecturas, proyectos, } \\
\text { ensayos, mapas conceptuales, grabaciones, ejercicios y problemas, búsquedas en } \\
\text { Internet etc.) }\end{array}$ & 3.09 & 1.363 \\
\hline La realización de exámenes, prácticas o trabajos de aplicación. & 3.05 & 1.266 \\
\hline El nivel de exigencia de mis profesores/as. & 2.86 & 1.304 \\
\hline Que me enseñen profesores/as muy teóricos/as. & 2.84 & 1.434 \\
\hline La poca claridad que tengo sobre lo que solicitan mis profesores/ as. & 2.84 & 1.293 \\
\hline No entiendo los temas que se abordan en la clase. & 2.67 & 1.419 \\
\hline $\begin{array}{l}\text { Mi participación en clase (conectarme a la clase, responder a preguntas, hacer } \\
\text { comentarios, desarrollar argumentaciones, etc.) }\end{array}$ & 2.61 & 1.381 \\
\hline La personalidad y el carácter de mis profesores/as que me imparten clases. & 2.57 & 1.242 \\
\hline Asistir o conectarse a clases aburridas o monótonas. & 2.52 & 1.588 \\
\hline Exposición de un tema ante la clase. & 2.51 & 1.367 \\
\hline La competitividad con o entre mis compañeros de clases. & 2.43 & 1.216 \\
\hline $\begin{array}{l}\text { Que mis profesores/as están mal preparados/as (contenido de la asignatura y/o } \\
\text { manejo de tecnología). }\end{array}$ & 2.04 & 1.509 \\
\hline
\end{tabular}


En general los estudiantes universitarios pueden experimentar altos niveles de estrés, ansiedad o depresión que puede influir en su bienestar personal y en su rendimiento académico (Aloufi et al., 2021), que es ocasionado por varios factores estresores por lo que se les debe prestar importancia, debido que el estrés académico es un factor que conlleva al desarrollo del riesgo suicida, especialmente en China que es un problema de salud pública (Ying et al., 2020); también se tiene estresores como la "ansiedad por el empleo y la ansiedad financiera" y "el apoyo de la universidad y del profesor" sobre la intención de abandono y el rendimiento académico de los estudiantes universitarios, así los estudiantes que tienen sistemas de apoyo fueron menos propensos abandonar los estudios que los que informaron de un menor apoyo por parte de su universidad y sus profesores (Noman et al., 2021).

Por otra parte el COVID-19 ha causado estragos en todas las economías, lo que ha provocado una enorme pérdida de empleo (Hossain, 2021), en consecuencia los estudiantes como medio para participar de las clases sincrónicas utilizan smartphone en reemplazo de una laptop o PC, por lo que se tiene mayor dependencia a los smartphones causando malestar psicológico (ansiedad general y depresión), considerado que el estrés académico está positivamente relacionado con la angustia psicológica, por lo que el estrés académico es un factor de riesgo para la dependencia de los teléfonos inteligentes, y pueden utilizar el teléfono inteligente en exceso como una forma de liberar la tensión cuando se enfrentan al estrés académico (Wang et al., 2020).

\section{Tabla 02}

Media de sintomas de estrés.

\begin{tabular}{lll}
\hline ITEMs & Media & $\begin{array}{l}\text { Desviación } \\
\text { estándar }\end{array}$ \\
\hline Fatiga crónica (cansancio permanente). & 3.09 & 1.32 \\
Dificultades para concentrarse. & 3.07 & 1.264 \\
Ansiedad (nerviosismo), angustia o desesperación. & 3.05 & 1.355 \\
Dolores de cabeza o migrañas. & 3.04 & 1.338 \\
Somnolencia o mayor necesidad de dormir. & 2.91 & 1.444 \\
Aumento o reducción del consumo de alimentos. & 2.89 & 1.307 \\
Inquietud (incapacidad de relajarse y estar tranquilo). & 2.88 & 1.268 \\
Sentimientos de depresión y tristeza (decaído). & 2.88 & 1.389 \\
Trastornos del sueño (insomnio o pesadilla). & 2.79 & 1.384 \\
Desgano para realizar las labores académicas. & 2.65 & 1.339 \\
Aislamiento de los demás. & 2.43 & 1.509 \\
Sentimientos de agresividad o aumento de irritabilidad. & 2.26 & 1.523 \\
Problemas de digestión, dolor de estómago o diarrea. & 2.18 & 1.414 \\
Rascarse, morderse las uñas, frotarse, etc. & 2.04 & 1.555 \\
Conflictos o tendencia a polemizar, contradecir, discutir o pelear & 1.93 & 1.493 \\
\hline
\end{tabular}


Como se tiene alta prevalencia de estrés en los estudiantes de la Escuela Profesional de Ingeniería en Industrias Alimentarias, en la Tabla 02 se muestra los síntomas como: fatiga crónica (3.09), continuado por las dificultades que tiene para concentrarse (3.07), que conlleva al estudiante a la ansiedad, angustia y desesperación (3.05), lo cual se refleja en dolores de cabeza o migrañas (3.04), que concuerda con otros estudios (Jerez-Mendoza \& Oyarzo-Barría, 2015). Estos aspectos son importantes debido a que puede causar problemas de desempeño académico como el bajo rendimiento debido al poco interés, desconcentración, falta de agilidad mental y vigor físico (Ayala Valenzuela et al., 2010).

\section{Tabla 03}

Media de las estrategias de afrontamiento ante el stress académico.

\begin{tabular}{|c|c|c|}
\hline ITEMs & Media & $\begin{array}{l}\text { Desviación } \\
\text { estándar }\end{array}$ \\
\hline Escuchar música o distraerme viendo televisión. & 3.22 & 1.345 \\
\hline Concentrarse en resolver la situación que me preocupa. & 3.08 & 1.189 \\
\hline Navegar en internet. & 2.99 & 1.251 \\
\hline $\begin{array}{l}\text { Mantener el control sobre mis emociones para que no me afecte lo que me } \\
\text { estresa. }\end{array}$ & 2.82 & 1.213 \\
\hline Evaluar lo positivo y negativo de mis propuestas ante una situación estresante. & 2.78 & 1.206 \\
\hline $\begin{array}{l}\text { Recordar situaciones similares ocurridas anteriormente y pensar en cómo las } \\
\text { solucioné. }\end{array}$ & 2.74 & 1.220 \\
\hline Fijarse o tratar de obtener lo positivo de la situación que preocupa. & 2.72 & 1.194 \\
\hline Búsqueda de información sobre la situación que me preocupa. & 2.69 & 1.322 \\
\hline $\begin{array}{l}\text { Habilidad asertiva (defender nuestras preferencias, ideas o sentimientos sin } \\
\text { dañar a otros). }\end{array}$ & 2.67 & 1.285 \\
\hline Hacer ejercicio físico. & 2.61 & 1.399 \\
\hline Establecer soluciones concretas para resolver la situación que me preocupa. & 2.61 & 1.170 \\
\hline Elogiar mi forma de actuar para enfrentar la situación que me preocupa. & 2.57 & 1.287 \\
\hline $\begin{array}{l}\text { Elaboración de un plan para enfrentar lo que me estresa y ejecución de sus } \\
\text { tareas. }\end{array}$ & 2.55 & 1.238 \\
\hline La religiosidad (encomendarse a Dios o asistir a misa) & 2.16 & 1.452 \\
\hline Contar lo que me pasa a otros. (verbalización de la situación que preocupa). & 2.06 & 1.341 \\
\hline Solicitar el apoyo de mi familia o de mis amigos. & 2.00 & 1.392 \\
\hline Jugar videojuegos. & 1.67 & 1.621 \\
\hline
\end{tabular}

En la Tabla 03 se muestra las estrategias de afrontamiento más utilizadas por los estudiantes es la audición de música, televisión (3.22), continuado por el empeño de concentrarse en resolver la situación que le preocupa (3.08) resultado similar a lo obtenido por Jerez-Mendoza \& Oyarzo-Barría, (2015) que menciona que el 89.8\% utiliza la estrategia de afrontamiento de "Concentrarse en resolver la situación que me preocupa", y navegar por internet (2.99) y las estrategias menos utilizadas fueron "jugar videojuegos", y "solicitar apoyo de mi familia o de mis amigos". 
Siendo necesario la implementación de estrategias a nivel institucional para prevenir los efectos del estrés como el bajo rendimiento académico, deserción estudiantil, siendo las técnicas utilizadas sobre el estrés académico la aromaterapia, utilizando aceite de lavanda (Ahmad et al., 2019), por ello es importante la implementación de programas de terapia forestal que disminuye la presión arterial sistólica y diastólica, considerando por los participantes la reducción del estrés (Rajoo et al., 2019), por lo que la universidad debe transitar hacia modelos más colaborativos y centrados en el estudiante (Pérez López et al., 2020), e implementar políticas para estabilizar este sistema respecto a la disponibilidad y la accesibilidad de la educación debido al COVID-19 (Dhanalakshmi et al., 2021), considerando que la calidad de la educación y la adquisición de conocimientos durante las actividades educativas virtuales son equivalentes o mejores que en las actividades presenciales tradicionales, por lo que el uso de la tecnología en las actividades educativas virtuales en un enfoque práctico puede ser conveniente para lograr los objetivos educativos deseados durante y potencialmente después de la pandemia de COVID-19 (Tuma et al., 2021).

\section{CONCLUSIONES}

Se tiene alta prevalencia de estrés académico en los estudiantes universitarios frente a la educación virtual que está asociada al COVID-19, siendo los factores estresores que producen mayor estrés académico en los estudiantes, la sobrecarga de tareas y trabajos que tienen que realizar diariamente, para cumplir en los plazos establecidos por los docentes para la presentación, así mismo el sistema de evaluación virtual, como consecuencia los estudiantes presentan síntomas como; la fatiga crónica, dificultad para concentrarse, ansiedad, angustia y desesperación, reflejado en dolores de cabeza o migrañas. Los estudiantes para afrontar buscan mecanismos de respuesta utilizando como estrategias: la audición de música, mirar televisión, navegar por internet en temas no relacionados aspectos educativos. Por lo que es importante la implementación de políticas universitarias como el plan de tutoría académica en los estudiantes universitarios que permitan reducir el estrés académico en los estudiantes.

\section{ORCID}

Olivia Magaly Luque Vilca: Universidad Nacional de Juliaca, Puno, Perú (DD

Nestor Bolivar Espinoza: Universidad Nacional de Juliaca, Puno, Perú (iD

Victor Ernesto Achahui Ugarte: Universidad Nacional de Juliaca, Puno, Perú (iD Perú (iD

Julio Rumualdo Gallegos Ramos: Universidad Nacional de Juliaca, Puno,

\section{FUENTE DE FINANCIAMIENTO}

El estudio fue autofinanciado.

\section{CONFLICTOS DE INTERÉS}

Los autores expresan que no existen conflictos de interés.

\section{AGRADECIMIENTO}

No aplica. 


\section{PROCESO DE REVISIÓN}

Este estudio ha sido revisado por pares externos en modalidad de doble ciego.

\section{DECLARACIÓN DE DISPONIBILIDAD DE DATOS}

No aplica.

\section{REFERENCIAS BIBLIOGRÁFICAS}

Ahmad, R., Naqvi, A. A., Al-Bukhaytan, H. M., Al-Nasser, A. H., \& Baqer Al-Ebrahim, A. H. (2019). Evaluation of aromatherapy with lavender oil on academic stress: A randomized placebo controlled clinical trial. Contemporary Clinical Trials Communications, 14(March), 100346. https://doi.org/10.1016/j.conctc.2019. 100346

Alania-Contreras, R. D., Llancari-Morales, R. A., Rafaele de La Cruz, M., \& OrtegaRévolo, D. I. D. (2020). Adaptación del cuestionario de estrés académico SISCO SV al contexto de la crisis por COVID-19. Socialium, 4(2), 111-130. https://doi. org/10.31876/sl.v4i2.79

AlAteeq, D. A., Aljhani, S., \& AlEesa, D. (2020). Perceived stress among students in virtual classrooms during the COVID-19 outbreak in KSA. Journal of Taibah University Medical Sciences, 15(5), 398-403. https://doi.org/10.1016/j.jtumed. 2020.07 .004

Aloufi, M. A., Jarden, R. J., Gerdtz, M., \& Kapp, S. (2021). Reducing stress, anxiety and depression in undergraduate nursing students: Systematic review. Nurse Education Today, 104877. https:// doi.org/10.1016/j.nedt.2021.104877

Ayala Valenzuela, R., Pérez Uribe, M., \& Obando Calderón, I. (2010). Trastornos menores de salud como factores asociados al desempeño académico de estudiantes de enfermería. Enfermería Global, 18, 1-13. https://doi.org/10.4321/s1695614120 10000100007

Baptista Lucio, P., Almazán Zimerman, A., \& Loeza Altamirano, C. A. (2020). Encuesta Nacional a Docentes ante el COVID-19. Retos para la educación a distancia. Revista Latinoamericana de Estudios Educativos, 50(ESPECIAL), 41-88. https:// doi.org/10.48102/rlee.2020.50.especial.96

Barraza A. (2007). Estrés académico: un estado de la cuestión. Psicología Científica.

Basturk, S. B., Dancer, C. E. J., \& McNally, T. (2020). Relationship of COVID-19 with Pregnancy. Pharmacological Research, 104743. https://doi.org/10.1016/j.tjog. 2021.03.005

Berrío García, N., \& Mazo Zea, R. (2011). Estrés Académico. Revista de Psicología Universidad de Antioquia, 3(2), 55-82.

Buzek, T., Poulain, T., Vogel, M., Engel, C., Bussler, S., Körner, A., Hiemisch, A., \& Kiess, W. (2019). Relations between sleep duration with overweight and academic stress-just a matter of the socioeconomic status? Sleep Health, 5(2), 208-215. https://doi.org/10.1016/j.sleh.2018.12.004 
Dhanalakshmi, R., Anuja Mary, A., Shrijith, D., \& Vijayaraghavan, N. (2021). A Study on Covid-19 - Impacting Indian Education. Materials Today: Proceedings, xxxx. https://doi.org/10.1016/j.matpr.2021.02.786

Gelineau-Morel, R., \& Dilts, J. (2021). Virtual Education during COVID-19 and Beyond. Pediatric Neurology, 119, 1-2. https://doi.org/10.1016/j.pediatrneurol.2021. 02.008

Gonzalez, L. (2020). Asociados a La Pandemia Por Covid-19. Artículo, ix(25), 158-179.

Hossain, M. (2021). The effect of the Covid-19 on sharing economy activities. Journal of Cleaner Production, 280, 124782. https://doi.org/10.1016/j.jclepro.2020.124782

Husky, M. M., Kovess-Masfety, V., \& Swendsen, J. D. (2020). Stress and anxiety among university students in France during Covid-19 mandatory confinement. Comprehensive Psychiatry, 102, 152191. https://doi.org/10.1016/j.comppsych. 2020.152191

IELSAC. (2020). COVID-19 y educación superior: De los efectos inmediatos al día después. Análisis de impactos, respuestas políticas y recomendaciones. Unesco, 1, 44-78.

Jerez-Mendoza, M., \& Oyarzo-Barría, C. (2015). Estrés académico en estudiantes del Departamento de Salud de la Universidad de Los Lagos Osorno. Revista Chilena de Neuro-Psiquiatría, 53(3), 149-157. https://doi.org/10.4067/s071792272015000 300002

Karim, M., Furnaz, S., Buksh, A. R., Beg, M. A., Khan, M. S., \& Moiz, B. (2013). Sample Size Calculation in Medical Research. MACS 2019 - 13th International Conference on Mathematics, Actuarial Science, Computer Science and Statistics, Proceedings, 2(8), 217-224. https://doi.org/10.1109/MACS48846.2019.9024807

Khosravani, V., Aardema, F., Ardestani, S. M. S., \& Sharifi Bastan, F. (2021). The impact of the coronavirus pandemic on specific symptom dimensions and severity in OCD: A omparison before and during COVID-19 in the context of stress responses. Journal of Obsessive-Compulsive and Related Disorders, 29(September 2020), 100626. https://doi.org/10.1016/j.jocrd.2021.100626

Noman, M., Kaur, A., \& Nafees, N. (2021). Covid-19 Fallout: Interplay between Stressors and Support on Academic Functioning of Malaysian University Students. Children and Youth Services Review, 106001. https://doi.org/10.1016/j.childyouth.2021. 106001

Pérez López, E., Vázquez Atochero, A., \& Cambero Rivero, S. (2020). Educación a distancia en tiempos de COVID-19: Análisis desde la perspectiva de los estudiantes universitarios. RIED. Revista Iberoamericana de Educación a Distancia, 24(1), 331. https://doi.org/10.5944/ried.24.1.27855

Rajoo, K. S., Karam, D. S., \& Abdul Aziz, N. A. (2019). Developing an effective forest therapy program to manage academic stress in conservative societies: A multi- 
disciplinary pproach. Urban Forestry and Urban Greening, 43(January), 126353. https://doi.org/10.1016/j.ufug.2019.05.015

Simonelli-Muñoz, A. J., Balanza, S., Rivera-Caravaca, J. M., Vera-Catalán, T., Lorente, A. M., \& Gallego-Gómez, J. I. (2018). Reliability and validity of the student stress inventory-stress manifestations questionnaire and its association with personal and academic factors in university students. Nurse Education Today, 64, 156-160. https://doi.org/10.1016/j.nedt.2018.02.019

Toribio-ferrer, C., \& Franco-Bárcenas, S. (2016). Estrés Académico: El Enemigo Silencioso del Estudiante. Salud y Administración., 3(200), 11-18.

Tuma, F., Nituica, C., Mansuri, O., Kamel, M. K., McKenna, J., \& Blebea, J. (2021). The academic experience in distance (virtual) rounding and education of emergency surgery during COVID-19 pandemic. Surgery Open Science, 1-5. https://doi. org/10.1016/j.sopen.2021.03.001

Wang, J. L., Rost, D. H., Qiao, R. J., \& Monk, R. (2020). Academic stress and smartphone dependence among Chinese adolescents: A moderated mediation model. Children and Youth Services Review, 118(April), 105029. https://doi.org/10.1016/j. childyouth.2020.105029

Ye, B., Wu, D., Im, H., Liu, M., Wang, X., \& Yang, Q. (2020). Stressors of COVID-19 and stress consequences: The mediating role of rumination and the moderating role of psychological support. Children and Youth Services Review, 118(September), 105466. https://doi.org/10.1016/j.childyouth.2020.105466

Ying, J., You, J., \& Guo, J. (2020). The protective effects of youth assets on the associations among academic stress, regulatory emotional self-efficacy, and suicidal risk: A moderated mediation model. Children and Youth Services Review, 119(October), 105660. https://doi.org/10.1016/j.childyouth.2020.105660

\section{CITAR COMO:}

Luque Vilca, O. M., Bolivar Espinoza, N., Achahui Ugarte, V. E., \& Gallegos Ramos, J. R. (2022). Estrés académico en estudiantes universitarios frente a la educación virtual asociada al covid-19. Puriq, 4, e200. https://doi.org/10.37073/puriq.4.1.200 